\title{
Rapid and Sensitive Detection of Nanomolecules by AC Electrothermal Flow Facilitated Impedance Immunosensor
}

\author{
Anil Koklu, *, Jason Giuliani, Carlos Monton ${ }^{3}$, Ali Beskok $^{4}$ \\ ${ }^{1}$ Biological and Environmental Science and Engineering, King Abdullah University of Science \\ and Technology (KAUST), Thuwal 23955-6900, Saudi Arabia \\ ${ }^{2}$ Department of Physics and Astronomy, the University of Texas at San Antonio, TX 78249, USA \\ ${ }^{3}$ General Atomics, P.O. Box 85608, San Diego, USA 92186 \\ ${ }^{4}$ Department of Mechanical Engineering, Southern Methodist University, Dallas, TX 75205, USA \\ *Corresponding author: anil.koklu@kaust.edu.sa
}




\begin{abstract}
Conventional immunosensors typically rely on passive diffusion dominated transport of analytes for binding reaction and hence, it is limited by low sensitivity and long detection times. We report a simple and efficient impedance sensing method that can be utilized to overcome both sensitivity and diffusion limitations of immunosensors. This method incorporates the structural advantage of nanorod-covered interdigitated electrodes and the microstirring effect of AC electrothermal flow (ACET) with impedance spectroscopy. ACET flow induced by a biased AC electric field can rapidly convect the analyte onto nanorod structured electrodes within a few seconds and enriches the number of binding molecules because of excessive effective surface area. We performed numerical simulations to investigate the effect of ACET flow on the biosensor performance. The results indicated that $A C$ bias to the side electrodes could induce fast convective flow, which facilitates the transport of the target molecules to the binding region located in the middle as a floating electrode. The temperature rise due to the Joule heating effect was measured using a thermoreflectance imaging method to find the optimum device operation conditions. The change of impedance caused by the receptors-target molecules binding at the sample/electrode interface was experimentally measured and quantified in real-time using the impedance spectroscopy technique. We observed that the impedance sensing method exhibited extremely fast response compared with those under no bias conditions. The measured impedance change can reach saturation in a minute. Compared to the conventional incubation method, the ACET flow enhanced method is faster in its reaction time, and the detection limit can be reduced to $1 \mathrm{ng} / \mathrm{ml}$. In this work, we demonstrate that this sensor technology is promising and reliable for rapid, sensitive, and real-time monitoring biomolecules in biologically relevant media such as blood, urine, and saliva.
\end{abstract}




\section{Introduction}

Immunoassays have been widely utilized as powerful and versatile biomedical tools for the diagnosis of certain food toxins $\mathbf{s}^{1,2}$, environmental pollutants ${ }^{3,4}$, or clinical diseases ${ }^{5-9}$. Because of the specific binding between receptors and target molecules, immunoassays have been broadly used to selectively bind biomolecules that are indicative of the presence of bacteria ${ }^{10}$, viruses ${ }^{11}$, or proteins ${ }^{12,13}$. Conventional immunoassays including the enzyme-linked immunosorbent assay (ELISA) are limited by their portability and high-cost operation because they rely on fluorescent/enzyme tagging and require sophisticated optical instruments. Recently, microfluidic integrated impedance spectroscopy immunosensors have become a common technique since it provides a label-free, low cost, portable, and sensitive detection for point of care applications ${ }^{14-16}$. In this technique, a small $A C$ voltage is applied to electrodes and, corresponding current and impedance are measured in a wide range of frequencies. For immunosensor applications, target molecule/receptor binding at the electrode surface induces alterations in the impedance, providing a direct means of detection on the sensor. However, the aforementioned techniques rely on receptors/ target molecules reactions, which take place through diffusion-dominated transport kinetics. Unfortunately, this mechanism is quite slow, resulting in long reaction time of several hours and low sensitivity. In extreme cases these reactions may not even occur. Thus, it is essential to develop a highly sensitive detection method for rapid screening and field-use applications varying from omics (genomics, proteomics) to nanoparticle (CRISPR-Cas9, exosomes, COVID-19) identification.

An effective way for increasing the sensitivity and, at the same time decreasing the response time, can be achieved by generating directional microflows that transport the target molecules toward functionalized sites. Several methods, such as hydrodynamic ${ }^{17,18}$, acoustic 19, 20 , and electrokinetic mixing ${ }^{21-24}$, have been employed to increase the reaction rate of receptors/target molecules binding. In the past few years, AC electro-kinetics/dynamics (ACEK-ACED) have been extensively used to manipulate analytes to the detection region ${ }^{25-33}$. All three major ACEK-ACED phenomena, dielectrophoresis (DEP) ${ }^{34-36}, A C$ electroosmosis (ACEO) ${ }^{37}$ and AC electrothermal $(A C E T)^{38}$, can be used to induce directional biomolecular movement. DEP force is directly applied over particles and is proportional to the cube of the target particle diameter. Brownian motion effects increasingly interfere with dielectrophoretic action of nanometer-sized particles, which results in weak attraction/repulsion forces ${ }^{39}, 40$. Moreover, DEP forces are short-ranged, and they are typically dominant within a few tens of microns away from the electrodes. It has been also reported that DEP does not work well in high conductivity media using planar surface electrodes 
41. The ACEO phenomenon typically occurs at low ionic strength, and ACEO flow velocity has been observed to decrease significantly with increasing electrolyte's conductivity and eventually becomes negligible at above $0.1 \mathrm{~S} / \mathrm{m}$, making it unsuitable for most bioassays ${ }^{42}$. Our previous studies on AC electrokinetics/electrohydrodynamics revealed that for a biologically relevant highconductivity media such as phosphate buffer saline (PBS, $\sigma \sim 1.4 \mathrm{~S} / \mathrm{m}$ ), ACET flow becomes the dominant phenomenon inducing directional and long-range convective vortices which can potentially drag the molecules to the middle of the electrodes' gap, then conveys them tangentially to the electrode surface of the interdigitated electrode pattern ${ }^{43,44}$.

The interdigitated electrode array is a well-known geometric configuration for ACET based microfluidic platforms, as it yields large electric field gradients at small applied potentials. Furthermore, the interdigitated electrode array offers favorable features in terms of low Ohmic drop, the fast establishment of steady-state, and increased signal to noise ratio ${ }^{45,46}$. Another important aspect of interdigitated electrodes is that microfluidic system can be designed to develop a robust electronic actuation system to perform a multiplexed protein assay. To carry out the multiplexed functionality, an array of different types of receptors can be patterned along a single microfluidic channel using a pair of addressable asymmetric interdigitated electrodes, where each element is targeting a specific target molecule. By selectively applying voltage at the terminals of each interdigitated electrode pair, ACET flow can assist transporting the target molecules toward the sensor surface so that the overall binding process can be accelerated. However, planar surface interdigitated electrodes fabricated by conventional photolithography methods have limited effective surface area due to nano-sized surface roughness ${ }^{24,25,33}$. Specifically, Cui et al. demonstrated the use of low voltage AC electrothermal effect to enhance and accelerate the detection of low abundance, IgG molecules by AC capacitive sensing with simultaneous ACET enrichment using interdigitated electrodes. After 30 seconds of measurement, the sensitivities of their device were obtained approximately $0.5 \%, 4 \%$ and $4 \%$ for $1 \mathrm{ng} / \mathrm{ml}, 10 \mathrm{ng} / \mathrm{ml}$, and $100 \mathrm{ng} / \mathrm{ml} \mathrm{lgG}$ samples, respectively. 3D nanostructured electrodes can be introduced to enhance sensor sensitivity because they increase the amount of the active binding sites, and consequently, the absorbed volume of target molecules ${ }^{47-50}$. Another fundamental limitation of usage of planar surface electrodes is the onset of electrode polarization (EP) that potentially overwhelms the impedance spectra at the low-frequency range $(<10 \mathrm{MHz})$ depending on electrode size ${ }^{51,52}$. Ions in the electrolyte move towards the electrode surface, leading to a massive interfacial impedance and causing a high-applied voltage drop, which decreases the sensitivity of the measurement. The most effective way to diminish the EP is to enlarge the electrode's effective surface area by depositing nanostructures on the electrode surface ${ }^{53-55}$. Well- 
ordered nanorod arrays with high density and aspect ratio seems to be the most suitable nanostructures due to their mechanical robustness and electrical stabilities and it can be fabricated on a large surface area using template assisted electrochemical deposition technique in a controllable manner ${ }^{56}$.

The significance of 3D nanostructured surfaces for immunoassay based current sensing was revealed by Lee et al using a field effect transistor configuration ${ }^{57}$. They utilized carbon nanotube networks decorated with gold nanoparticles, which acted as the actual attaching sites for the probe molecules. The gold nanoparticle decorated carbon nanotube network was fabricated between the island electrode (drain) and enclosing electrode (source) and used as a channel where the antibody/antigen binding takes place and consequently, it changes the current flowing from drain to source. For $100 \mathrm{nM}$ target molecules, they have experimentally shown that the sensitivity of their device was enhanced for the AC biased case ( 23\%) compared to the unbiased case $(\sim 16 \%)$ after 10 minutes operation at $1 \mathrm{MHz}-5 \mathrm{~V}$ condition. However, maintaining a relatively low temperature rise at these operation conditions is a major challenge for high ionic strength solution $(1 \mathrm{~S} / \mathrm{m})$ since higher conductivity electrolyte are more susceptible to Joule heating effects. Thus, the ACET-induced self-heating must be characterized prior to any electric field excitation to avoid unwanted effects and ensure that ACET is applicable and biologically safe for the required applications. For this purpose, thermoreflectance imaging method was integrated to the fluidic platform to measure the temperature rise at the electrode/electrolyte interface in our previous article. It provides experimental temperature determination in ACET-based microfluidic applications, which allows one to tailor the sensor geometry to keep the Joule heating effects within acceptable ranges for biological samples, or other temperature-sensitive applications ${ }^{43}$.

The hydrophilicity of the sensor surface is a prerequisite for creating a high binding affinity receptor region. In most cases, the surface of the hydrophilic substrate, where the sensors are held, is conjugated with receptors, resulting in low quantities of binding cites on the sensor region. This is due to adhesive forces which cause the receptor assays to spread out over the sensor region ${ }^{57}$. Therefore, localization of the receptors on an electrode surface is the key parameter to fabricate a high-performance immunosensor. This can be achieved by hybrid surfaces at which the electrode surface presents hydrophilic characteristic, while the rest of the substrates remain hydrophobic. Lots of low surface energy materials were selected to modify a hydrophilic surface, such as tridecafluorooctyltriethoxysilane, heptadecafluorodecyl trimethoxysilane, actadecyltrichlorosilane, n-octadecanethiol, self-assembly of alkanoic acid through a solutionimmersion process, and hexamethyldisilazane (HMDS) as the silylation reagent. A facile, inexpensive, and general approach is explored for the fabrication of transparent hydrophobic 
surface using HMDS ${ }^{58}$. One unique aspect of HMDS compared with many other passivation layers is that it can react with the surface of $\mathrm{SiO}_{2}$ or glass to form a self-assembled monolayer and transform the hydrophilic surface to hydrophobic ${ }^{59}$. HMDS can also exhibit a surface roughness comparable to that observed for two-dimensional materials supported by glass substrate $^{60}$. Furthermore, it shows highly biocompatible characteristics which makes it a good candidate for biosensor applications ${ }^{61,62}$.

In this study, we present an effective impedance-based sensing strategy that can significantly enhance speed and sensitivity by combining the structural advantages of nanorod electrodes fabricated on a hydrophobic substrate while inducing ACET flow to accelerate the transport of analyte toward the sensing region with immobilized receptors. In this approach, the target molecule level was simultaneously quantified by monitoring the impedance change because of the specific binding of target molecules with receptors. We used numerical simulations to verify that the ACET flow can assist the transport of the target molecules toward the sensor surface so that the overall binding process can be accelerated. Because of the electrokinetic enhancement, the detection sensitivity could be enhanced by at least one order of magnitude (compared to the ELISA kit) to reach a nanogram per milliliter ( $\mathrm{ng} \mathrm{m}^{-1}$ ) level, and the detection time could be reduced from several hours to a few seconds. This paper is organized as follows: we first present the sensor fabrication, buffer preparation and sensor surface functionalization, impedance spectroscopy technique, numerical simulations, and the detection mechanism, respectively. Then, experimental results are discussed. Finally, conclusions are presented.

\section{Materials and Methods}

\section{Sensor Fabrication and Surface Modification}

Microfabrication fabrication process is extensively explained in supporting information and summarized in Figure S-1. Briefly, after cleaning the glass substrates, they were vertically dipped into a beaker which was filled with HMDS and acetone solution with 1:1 ratio for various durations. The variation of surface wettability depending on the dipping duration, was explained in the supporting information (Figure S-2). Then, the interdigitated electrode array was fabricated on HMDS coated glass substrates using standard photolithography method. The nanopatterning of electrode surfaces was carried out using the template-assisted electrodeposition method, and the details of fabrication were reported in ${ }^{56}$. A nanoporous anodic aluminum oxide (AAO), which was used as a template, was placed on the planar gold electrode surface and then nickel and gold 
were sequentially deposited electrochemically through the nanopores. (Figure 1a, 1b, and 1c).

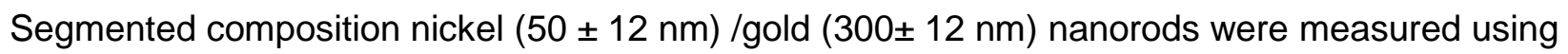
SEM images taken at a 45 degrees inclination at various locations on the electrode. The diameter of the nanorods were determined by using a microscopy image segmentation tool (MIST) and is obtained as $102 \pm 12 \mathrm{~nm}$. The aspect ratio (height/diameter) of nanorod is calculated as 3.43 which provides self-standing nanorods structures. The tailored nanorods' aspect ratio enables us to maximize the effective receptor/target molecule binding region. After electrochemical deposition, the AAO template was removed in a chromic-phosphoric acid solution at room temperature for 60 minutes.
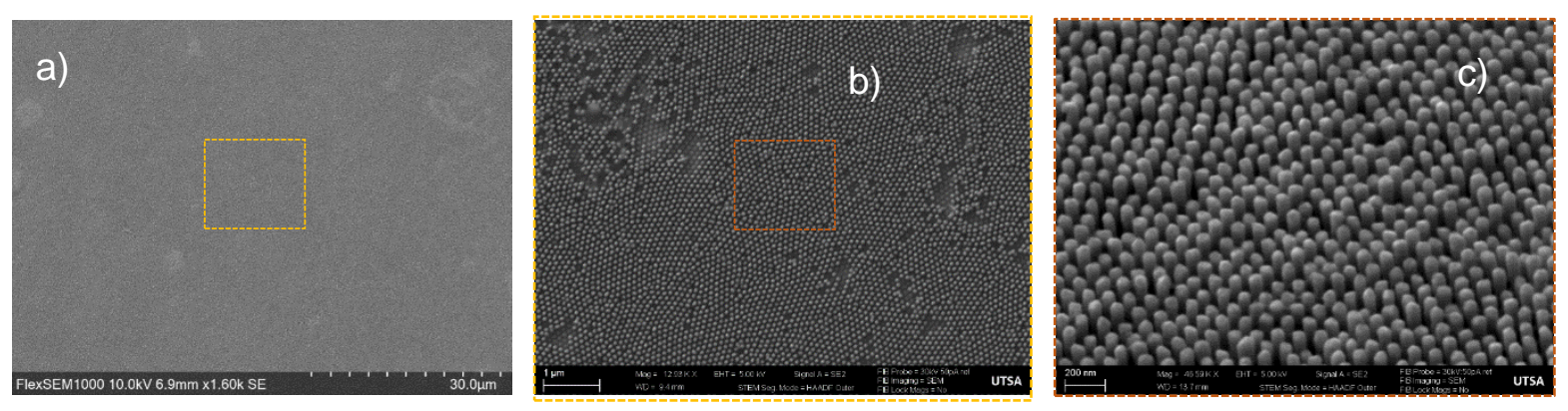

Figure 1. SEM analysis of the nanorod patterned electrodes. (a), and (b) show the top view of the array of nanorods at different magnifications. (c) Tilted view of the nanorods.

Ultra Violet (UV) treatment is an excellent method for increasing the surface hydrophilicity without influencing the electrode microstructure characteristics ${ }^{63}$. The HMDS part of the substrate was covered with a photomask to preserve its hydrophobic behavior, but the electrodes were exposed to UV for 10 minutes at the power of $110 \mathrm{~mJ} / \mathrm{cm}^{2}$. By doing this, receptors can be selectively coated on the electrode surface which reduces the number of target molecules potentially binding to the other surfaces.

Electrodes with larger sizes were considered to be more cooperative to induce AC electrothermal flow and were successfully used to detect small molecules ${ }^{33}$. Therefore, the device consists of three interdigitated electrodes with $200 \mu \mathrm{m}$ width and $200 \mu \mathrm{m}$ spacing. A rectangular portion of the tape was cut using a craft cutter to create a micro-channel with $0.4 \times 10 \times$ $25 \mathrm{~mm}$ (height $(H) \times$ width $(W) \times$ length $(L)$ ) dimensions. The wires used for electrical connections were bonded using conductive silver epoxy (MG Chemicals). Another HMDS coated glass slide was used as a cover on the top of the microfluidic channel to increase the flow velocity due to velocity slip on HDMS surfaces and reduce the possibility of target molecules binding. A 
diamond drill was used to create the fluidic ports, and copper tapes were used for electrical connections.

\section{Buffer Solution Preparation and Electrode Surface Functionalization}

PBS $(\sigma \sim 1.4 \mathrm{~S} / \mathrm{m})$ was prepared by $1: 10$ volume dilution of physiological strength stock solution with deionized water to obtain $1 \mathrm{mM}$ phosphate buffer $(\mathrm{pH} 7.0)$ containing $0.05 \mathrm{v} / \mathrm{v} \%$ Tween 20 (Fisher Scientific). The sensitivity of the microfluidic sensor was determined by measuring the binding of goat anti-bovine IgG $(\mathrm{H}+\mathrm{L})$ (Jackson ImmunoResearch Laboratories Inc.) (Receptors) to bovine IgG whole molecules (Jackson ImmunoResearch Laboratories Inc.) (Target molecules). Bovine $\lg G$ whole molecules were immobilized on the electrodes prior to the test. The microfluidic chip was incubated in an incubator for different time scales ranging from $1 \mathrm{~h}$ to $6 \mathrm{~h}$. Different concentrations of the anti-bovine $\lg G$ antibody were loaded at concentrations ranging from 1 to $100 \mathrm{ng} / \mathrm{ml}$ in PBS.

\section{Detection Mechanism}

The detection mechanism is based on interrogating the impedance change at the interface between a sensor electrode and the sample solution due to the binding reaction. Figure 2 schematically shows the operation mechanism of this sensor. An AC voltage at a certain frequency was applied to the side electrodes using a function generator (Tektronix AFG3102). ACET flow carries the target molecules to the immobilized receptors region and the impedance simultaneously increases due to the chemical binding. Then, impedance measurements were conducted by switching the input from function generator to impedance analyzer (4194A, Agilent) via an electrical switch for every 7.5 seconds which gives 8 data points for every minute. First, the impedance analyzer was operated at 401 discrete frequencies from $1 \mathrm{kHz}$ to $10 \mathrm{MHz}$ at 20 $\mathrm{mV}$ to determine the optimum frequency range for molecule detection. The impedance measurements and equivalent circuit model (Figure S-3) analysis were explained in supporting information. The impedance spectra of PBS solution were measured before and after the receptors coating. When the optimum frequency was selected, impedance measurements were performed at a single frequency for the target molecule detection. The impedance of the system spontaneously increases until all the target molecules are trapped on the functionalized site of the electrodes. The binding of target molecules to the receptors can be obtained by calculating the normalized impedance change rate using the following equation, 


$$
\Delta Z / Z_{\text {reference }}=\left(Z_{\text {after binding }}-Z_{\text {before binding }}\right) / Z_{\text {before binding }}
$$

The calculated $\Delta Z / Z_{\text {reference }}$ was used as an indication of target molecules binding to the antibody.

A theoretical model describing ACET flow facilitated antibody/antigen reaction in microfluidic system was extensively explained in the supporting information. The model consists of electrostatic, energy, Stokes, and first order Langmuir adsorption model coupled with transport equations.
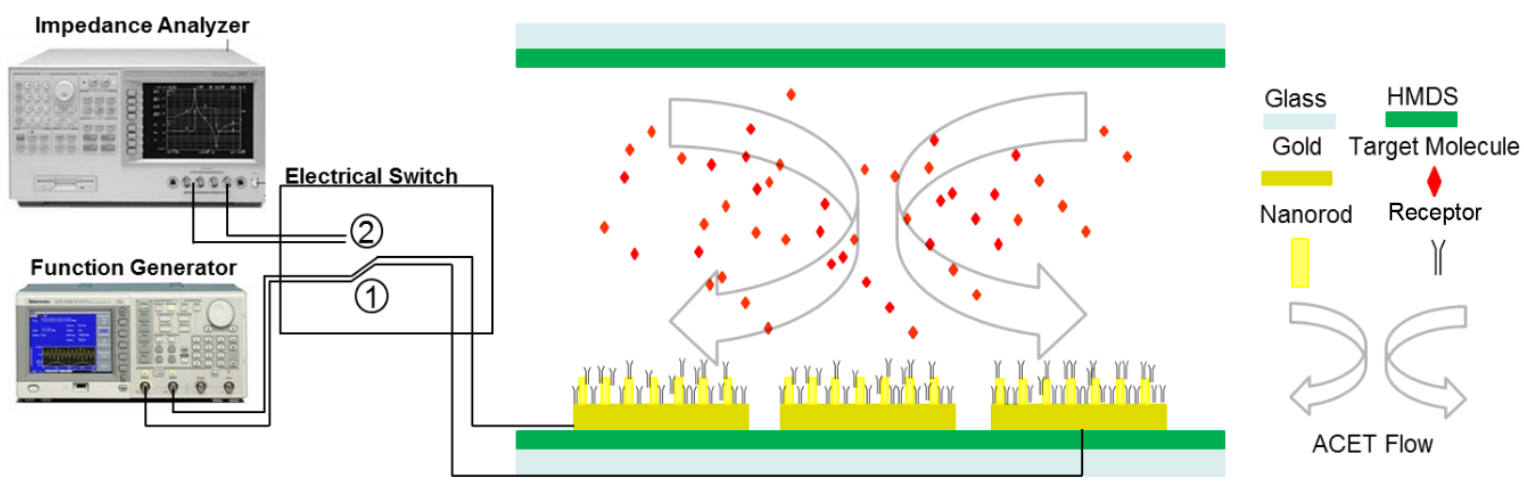

Figure. 2 Configuration of the experimental setup. A function generator supplied an AC potential to induce ACET flow to concentrate target molecules onto the receptors-immobilized gold nanorod electrodes, and the binding was then measured using an impedance analyzer for the quantification of the analyte.

\section{Results}

The importance of wettability for impedance measurements

Wetting characteristics of the electrodes were investigated utilizing contact angle measurements. Static contact angle measurements were explained in the supporting information. The baseline glass surface shows a hydrophilic characteristic with a water contact angle of $\sim 19 \pm 2.3^{\circ}$. After the surface of the glass was treated for $24 \mathrm{~h}$ in HMDS solution, the surface became hydrophobic with a water contact angle of $99^{\circ} \pm 1.7$. The change of the wettability characteristic is attributed to the chemistry of the HMDS. Bare glass is hydrophilic because of $\mathrm{OH}^{-}$termination and water molecules can easily attach to the hydrogen of these silanol groups on the $\mathrm{SiO}_{2}$ to form a thin water film but HMDS screens water molecules. After coating the glass surface with HMDS for 24h, gold interdigitated electrodes were fabricated using standard photolithography method described in the sensor fabrication part (Figure S-4a). The contact angle of a water droplet on the 
gold electrode was measured $77.87^{\circ} \pm 1.54^{\circ}$ (Figure $\mathrm{S}-4 \mathrm{~b}$ ). After 10 minutes of selectively exposing a UV using mask aligner (MJB-3-Mask Aligner) at the power of $150 \mathrm{~W}$, the water droplet completely infiltrated into the electrode surface, indicating a marked surface transition from hydrophilic to super hydrophilic, resulting in a contact angle less than $10^{\circ}$ for planar electrodes (Figure S-4c).
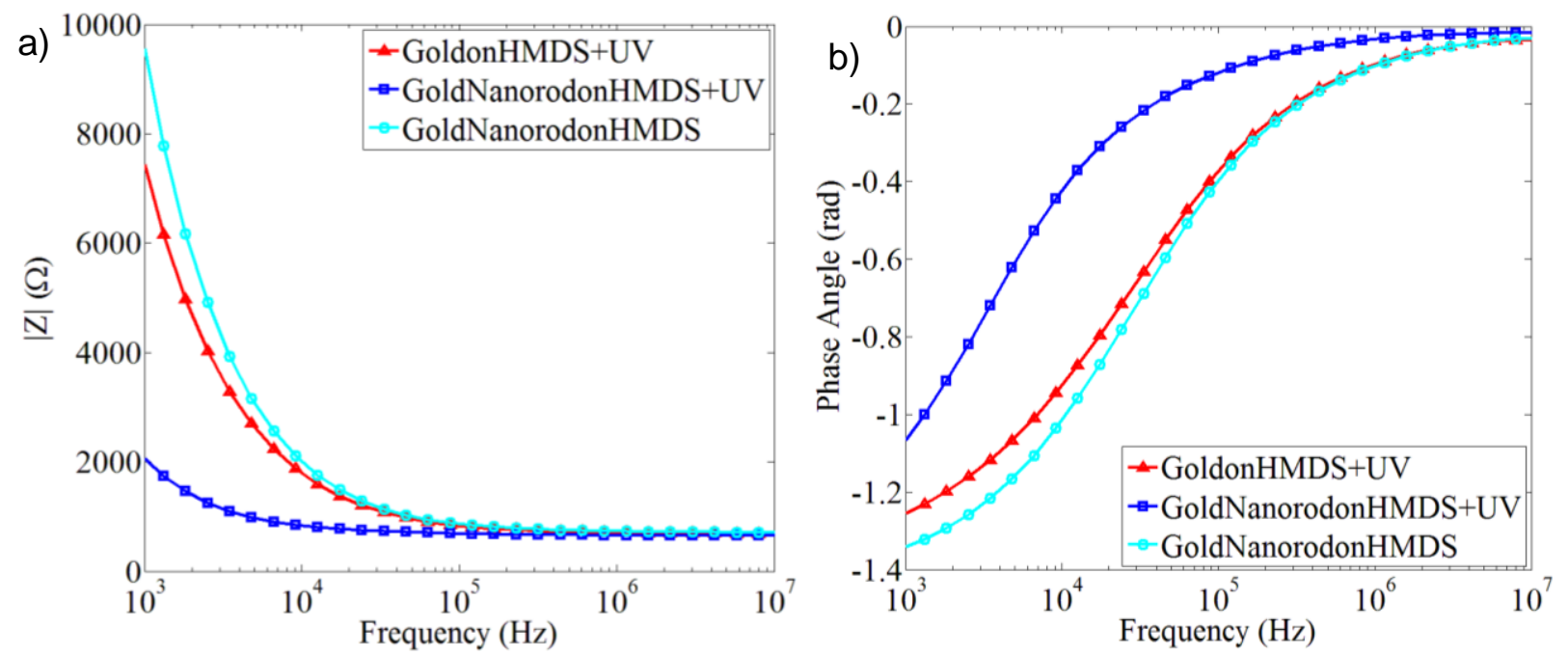

Figure 3. Impedance magnitude (a) and phase angle (b) spectrum of gold planar (red) and gold nanorod structured (dark (After UV treatment) and light blue (Before UV treatment)) electrodes.

It is well-known that vertically oriented nanorod with the well-faceted (001) shows superhydrophobic characteristics because of high surface roughness and low surface free energy but we could not conduct contact angle measurements due to the small actual electrode area (Figure S-1, Red dashed rectangle). However, the hydrophobic behavior of the nanorod structured electrodes can be indirectly assessed by impedance measurements. The impedance measurements were performed with PBS for nanorod and planar electrodes. Each measurement was repeated at least three times, and the average impedance magnitude and phase angles were reported. The normalized standard deviation in all cases was less than $1 \%$ and hence, they were not shown in the figure for the sake of clarity. Figure 3 shows the Bode representation of the average impedance spectra of gold planar and nanorod structured electrodes. At frequencies below $1 \mathrm{MHz}$, the impedance magnitude increased with decreasing frequency, and the phase angle value shifted toward $-\pi / 2$ radians. These changes are revealing capacitive charging at the electrode surface because of the EP effect. Since this phenomenon is mainly capacitive $(C)$, it is 
proportional to the surface area of electrodes. As the effective surface area $(A)$ of the electrode increases with nanorod structures, the magnitude of the impedance should proportionally decrease $(Z \sim 1 / C)$ but the results were not consistent with the impedance behavior of a capacitor. Nanorod structured surfaces tended to be more hydrophobic, leading to poor reaction kinetics between the electrolyte and the electrode and weakening the ionic transport. Bubble pockets can shield the electrode surface which causes a high impedance value. After the nanorod electrode surface was exposed to UV, the impedance magnitude decreased drastically compared to that of measured with a gold planar electrode and the usable frequency bandwidth expanded (Figure 3).

Selection of sensor operation conditions

To ensure good electrical connections and proper impedance behaviors of the electrodes, before the ACET activation, the impedance spectra of the sensor filled with PBS were measured from 1 $\mathrm{kHz}$ to $10 \mathrm{MHz}$ with an excitation voltage of $20 \mathrm{mV}$ in the absence of target molecules. In the lowfrequency range, EP overshadowed the impedance spectra and this behavior was diminished about $5 \mathrm{MHz}$ for gold planar electrodes and the impedance magnitude converges a frequencyindependent resistive plateau (Figure 3). Therefore, $5 \mathrm{MHz}$ was selected as a critical frequency for the impedance-based sensitivity measurements.

The ACET force expression can be expressed in terms of a directional parameter $M=$ $\left(\left(\frac{T}{\sigma} \frac{\partial \sigma}{\partial T}-\frac{T}{\varepsilon} \frac{\partial \varepsilon}{\partial T}\right) /\left(1+(\omega \tau)^{2}\right)\right)+0.5 \frac{T}{\varepsilon} \frac{\partial \varepsilon}{\partial T}$, which is a function of frequency for a fixed type of liquid and temperature (Parameters are defined in supporting information). The amplitude of ACET force can be predicted with $M$ factor, which remains maximum until $10 \mathrm{MHz}$ for $1.4 \mathrm{~S} / \mathrm{m}$ PBS solution (Figure S-5) and therefore, the side electrodes were also excited at $5 \mathrm{MHz}$ using the function generator. Moreover, most of the AC potential would be applied over the resistive fluid bulk which poses the highest $\mathrm{AC}$ electrothermal flow velocities ${ }^{43}$.

The amplitude of applied potential cannot be increased arbitrarily because higher voltages may lead to detachment of the functionalized layer over the electrode surface and lead to malfunction of the sensor due to the Joule heating effect. At high conductivities, temperatures beyond a few degrees $\left(T>10^{\circ} \mathrm{C}\right)$ are likely to occur under an applied $A C$ potential of even a few volts ${ }^{64,65}$. Therefore, the ACET-induced temperature must be measured before any electric field excitation to avoid unwanted effects and ensure that ACET is applicable and biologically safe for the required applications $24,33,66,67$. The temperature rise at the electrodes induced by Joule heating is characterized using the TMX Scientific $\mathrm{T}^{\circ}$ Imager ${ }^{\circledR}$ thermal-reflectance (TR) imaging system. TR method, which is based on measuring the temperature induced surface reflectance change, 
is an optical thermal metrology method that is nondestructive, noninvasive, and noncontact. The method employs a lock-in differential measurement that improves its precision, and uses monochromatic light illumination in the visible spectrum, which can provide the spatial resolution to around $0.2-0.5 \mu \mathrm{m}$ and detect $0.1 \mathrm{~K}$ temperature changes. It also does not require special sample preparations, can obtain quick thermal maps, and can optically reach the electrode regions in microchannel. The detail of the measurement technique is explained in Ref ${ }^{43}$ and supporting information. As expected from the Joule heating term, temperature increases with increasing activation voltage, and hence, the temperature rise was measured at different excitation voltages at $5 \mathrm{MHz}$. The results are given in Figure S-6 in supporting information. The temperature rise was obtained less than $10{ }^{\circ} \mathrm{C}$ up to $10 \mathrm{~V}_{\mathrm{pp}}$ and is considered safe for biomicrofluidic applications.

The importance of wettability for surface functionalization

The fast binding reactions were considered between the receptors and electrodes due to the hydrophilicity of the UV exposed electrode surface. After the surface of electrodes was treated with the receptors inside an incubator, it was gently rinsed with PBS and the microfluidic channel was placed by centering the electrodes in the middle. Then, impedance measurements were conducted by pipetting PBS buffer in the microfluidic channel.

After 6 hours of incubation process, impedance values have increased from $688.2 \Omega$ ( 0 hours) to $723.4 \Omega$ (6 hours) at $5 \mathrm{MHz}$ for UV treated planar gold electrodes, indicating adequate molecular immobilization on the electrode surface. The importance of a hybrid surface to increase the concentration of receptors on the electrode surfaces was evaluated by comparing the impedance spectra measured with the gold planar electrode fabricated on a hydrophilic glass surface. The impedance behavior of PBS measured with an hour incubated gold planar electrode fabricated on the glass surface $\left(1 \mathrm{~h}^{*}\right)$ was obtained similar to that of gold electrodes fabricated on HMDS at 0 -hour incubation. Since the receptor solution spreads out on the glass surface, the concentration of receptors immobilized on the electrode is very low to induce a distinction on the impedance spectra. Then, the same measurements were repeated for UV treated gold nanorod structured electrodes fabricated on HMDS coated glass slides. The impedance spectra are shown in Figures 4c and $4 \mathrm{~d}$. First, the impedance magnitude increased from $605.6 \Omega$ to $945.1 \Omega$ at $5 \mathrm{MHz}$ after an hour incubation with the receptors.

The reason for this huge difference can be attributed to the area of the active binding regions, which is increased in the presence of nanorods. The second important point is the saturation of 
the attached receptors after an hour incubation because the impedance magnitude remained almost the same for the case of 6-hour incubation.
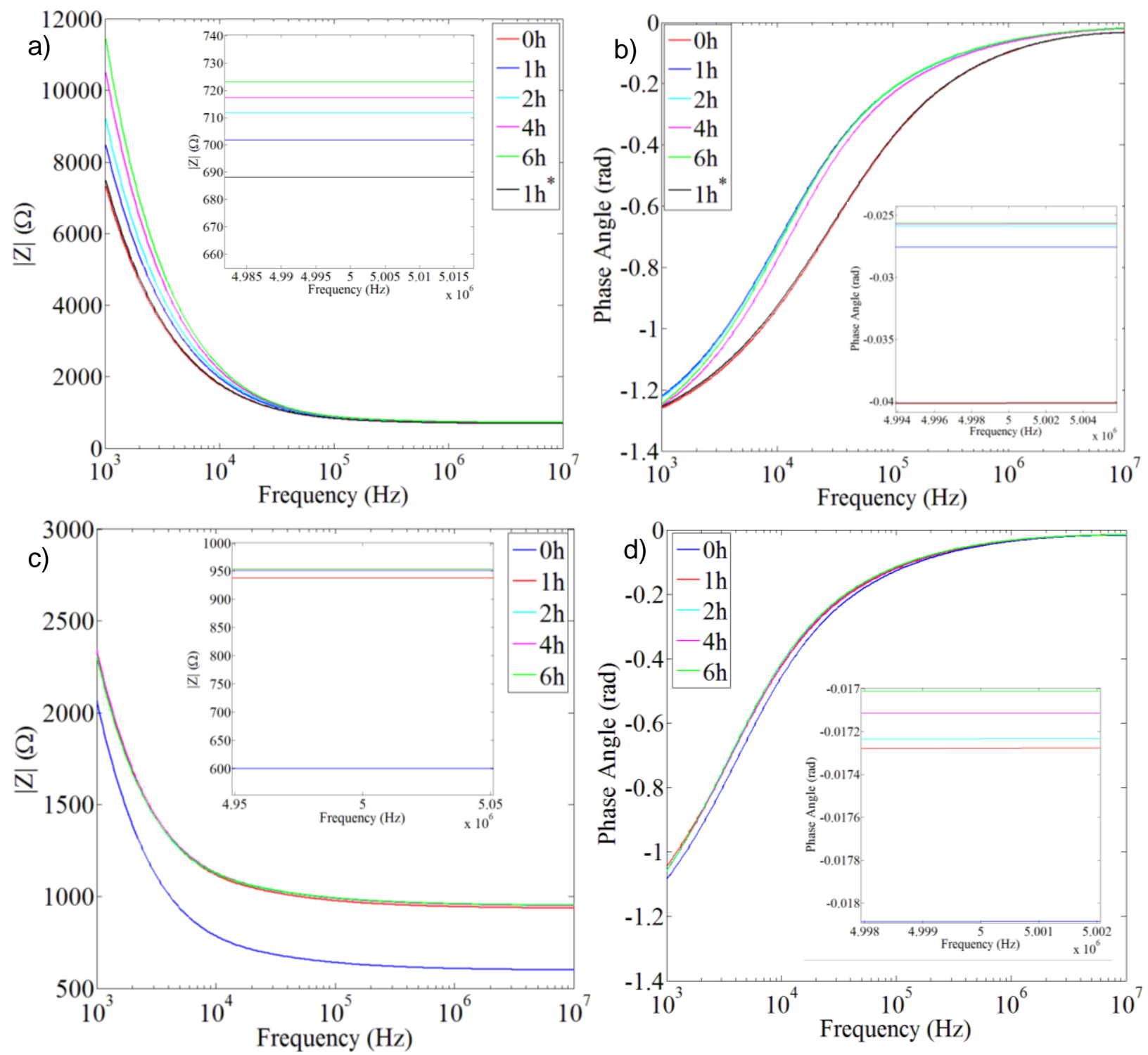

Figure 4. Impedance magnitude (a) and phase angle (b) for gold planar electrode fabricated on HMDS for different antibody incubation times ranging from 0 to 6 hours. Black line represents the impedance spectra of receptors incubated on gold electrode fabricated on glass for an hour. Impedance magnitude (c) and phase angle (d) for gold nanorod electrode at different antibody incubation times ranging from 0 to 6 hours.

\section{Target Molecule Detection}

The applied voltage range was determined using the thermoreflectance method explained in the "Selection of sensor operation conditions" section but the effect of applied voltage on sensitivity 
measurements must be investigated by sweeping the voltage from 1 to $10 \mathrm{Vpp}$. The side electrodes were excited with $180^{\circ}$ phase difference for 60 seconds, and the results are depicted in Figure 5 for UV treated planar and nanorod electrodes. In the absence of ACET flow, the microfluidic devices could not sense the target molecules in a minute because of slow diffusionbased transport $\left(t_{D} \approx \frac{L^{2}}{D} \sim 30\right.$ minutes).

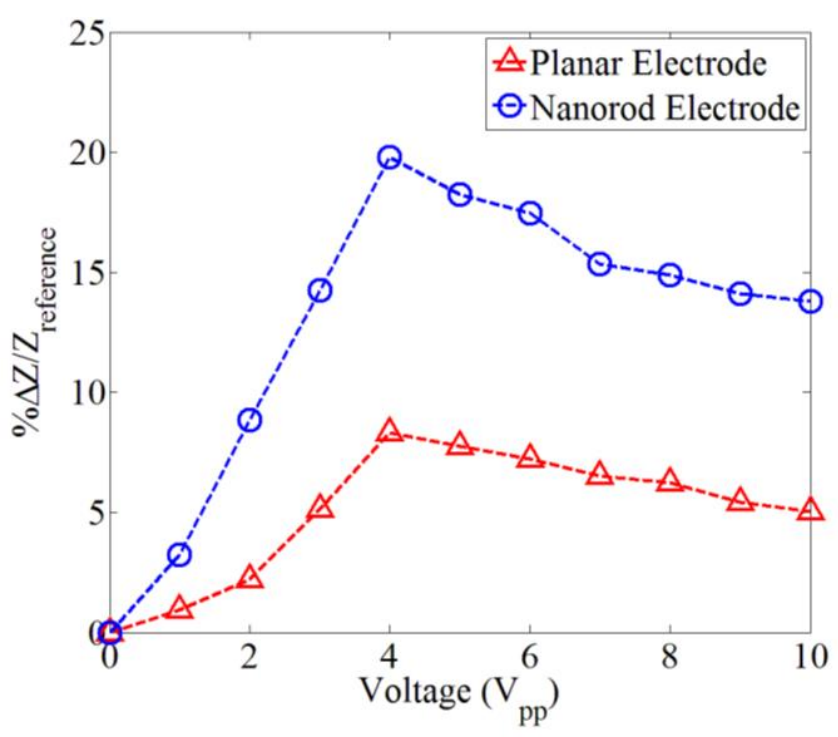

Figure 5. Percentile normalized impedance change with different applied voltages for $1 \mathrm{ng} / \mathrm{ml}$ concentration of target molecules mixed PBS solution for 60 seconds.

On the other hand, they were transmitted onto the sensor region via the circulatory flow and trapped on the receptors. The normalized impedance-change increases until a critical applied voltage $\left(4 \mathrm{~V}_{\mathrm{pp}}\right.$ ), then it drops to a certain value. The possible reasons are the low residence time of target molecules on receptors or the degradation of receptors due to temperature rise. Although high applied voltages $\left(F_{E T} \sim V^{4}\right)$ can induce fast convection flow, which facilitates the transport of the target molecules to the binding region; they could not find enough time to attach to the immobilized receptors. Moreover, temperature rise can potentially cause damage to the receptors which decreases the number of captured target molecules on the sensor.

The specific binding reactions were conducted with Bovine lgG whole molecules by applying a 5 $\mathrm{MHz} A \mathrm{AC}$ voltage at $4 \mathrm{~V}_{\mathrm{pp}}$ for $\mathrm{ACET}$ flow, which increases the temperature by $2^{\circ} \mathrm{C}$ on the electrode surface (Figure S-6). $5 \mathrm{MHz}$ AC signal at $20 \mathrm{mV}$ was employed as the measuring signal. Electrodes with 6 hours of incubated receptors were used for these measurements. The normalized impedance change rates were plotted as a function of time in Figure 6 for planar 
(Figure 6a) and nanorod structured (Figure 6b) electrodes using three different concentrations of the solution.
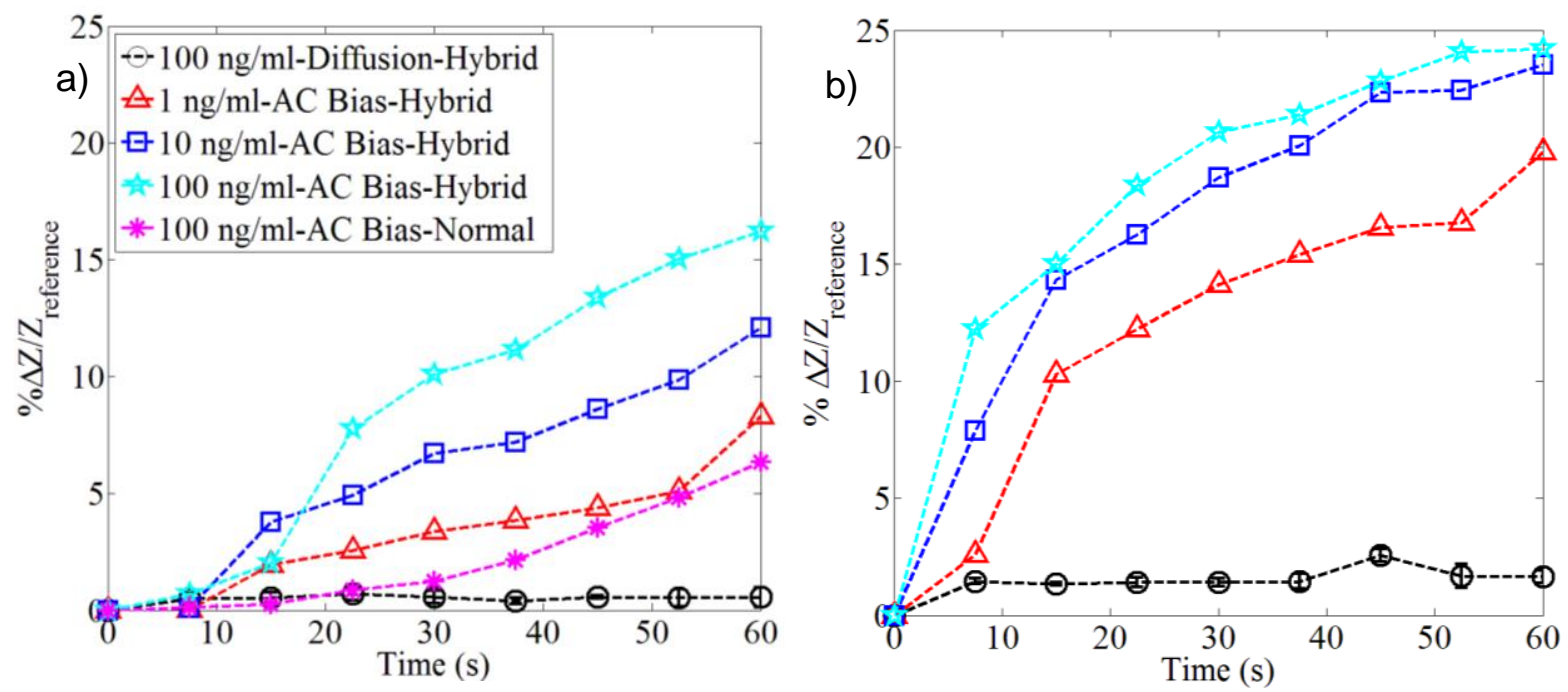

Figure 6. Percentile normalized impedance changes with time for different concentrations of target molecules measured by planar (a) and nanorod (b) electrodes. The ACET flow is generated by applying $5 \mathrm{MHz} A C$ voltage at $4 \mathrm{~V}_{\mathrm{pp}}$ and $5 \mathrm{MHz} A C$ signal at $20 \mathrm{mV}$ was employed as the measuring signal.

As shown in Figure 6, the impedance of the solution without ACET flow remains constant around zero during the test. However, the impedance of the system with ACET flow increases monotonically with time due to the binding reactions. AC biasing led to a significant enhancement of the binding rate compared with that under unbiased conditions. For $100 \mathrm{ng} / \mathrm{ml}$ of target solution, the impedance change reached almost $15 \% / \mathrm{min}$ and $25 \% / \mathrm{min}$ within the 60 s using the planar and nanorod structured electrodes, respectively. The impedance change rates for various dilutions of target molecules are also demonstrated in Figure 6. As expected, when the sample became more diluted, the impedance change decreased to $8 \% / \mathrm{min}$ and $20 \% / \mathrm{min}$ for planar and nanorod electrodes. The results in Figure 6 demonstrate that ACET facilitated impedance method is a quantitative method within a detection range of $1 \mathrm{ng} / \mathrm{ml}$ of target molecules.

The binding process was also simulated using COMSOL Multiphysics for the same size of device and concentration of molecules to visualize the binding reaction better. Unless the analyte solution is moved towards the sensor surface using an external force, a few microns thick analyte depletion zone forms next to the sensor surface in the solution, which causes poor detection and low sensitivity in biosensing (Figure S-7a). After the side electrodes were excited at $4 \mathrm{~V}_{\mathrm{pp}}$, the thickness of the depletion zone was significantly increased, which indicated that the depletion of 
the target molecules near the sensor surface was suppressed as a result of the continuous supply of the target molecules from the bulk electrolyte (Figure S-7b).

Conclusion

The electrodes in this work were planar and nanorod structured interdigitated gold electrodes that are fabricated by standard photolithography and template-assisted electrochemical deposition method. Using hybrid surfaces reduces the pumping power and most importantly allows functionalization of the sensor surfaces with antibodies, which not only minimizes the amount of used antibodies but also decreases cross-contamination between distinctly functionalized sensor surfaces. The binding process was aided by AC electrothermal flow that accelerates the transport of target molecules to the electrodes, where the surface was functionalized with receptors and enhance the detection of target molecules. The changes in the impedance at an optimum frequency $(5 \mathrm{MHz})$ were measured every $7.5 \mathrm{~s}$ within a minute. The impedance change reached almost $15 \% / \mathrm{min}$ and $25 \% / \mathrm{min}$ within the 60 s using the planar and nanorod structured electrodes, respectively. The results demonstrate that the ACET enhanced impedance immunosensor allows the directed immobilization of molecules onto microelectrodes at a very low concentration of $1 \mathrm{ng} / \mathrm{ml}$ in a significant short time (the 60s). The ACET flow integrated impedance immunosensors will greatly expand the application scope of electric field driven detection methods in microfluidic chips. In the future, a microfluidic chip with an asymmetric interdigitated electrode array, where each pair of electrode's surface-functionalized with different receptors, will be fabricated and integrated with a robust electronic actuation system to perform as a multiplexed immunosensor. The physiological media will be circulated in a loop utilizing ACET flow and the change in the impedance due to the binding of target molecules and receptors will be simultaneously monitored for each electrode pair.

Supporting Information

Electrode fabrication process, contact angle measurements, impedance spectroscopy measurements, M-factor calculation, temperature rise measurements, theory and numerical simulations.

\section{Acknowledgements}

The authors are grateful to Mr. Assaad El Helou and Dr. Peter E. Raad for their assistance to integrate thermoreflectance method to fluidic systems. We are also thankful to SMU Hunt Institute for their support. 


\section{References}

1. Raeisossadati, M. J.; Danesh, N. M.; Borna, F.; Gholamzad, M.; Ramezani, M.; Abnous, K.; Taghdisi, S. M., Lateral flow based immunobiosensors for detection of food contaminants. Biosensors and Bioelectronics 2016, 86, 235-246.

2. Orlov, A. V.; Khodakova, J. A.; Nikitin, M. P.; Shepelyakovskaya, A. O.; Brovko, F. A.; Laman, A. G.; Grishin, E. V.; Nikitin, P. I., Magnetic immunoassay for detection of staphylococcal toxins in complex media. Analytical chemistry 2013, 85 (2), 1154-1163.

3. Lafleur, J. P.; Senkbeil, S.; Jensen, T. G.; Kutter, J. P., Gold nanoparticle-based optical microfluidic sensors for analysis of environmental pollutants. Lab on a Chip 2012, 12 (22), 4651-4656.

4. Pol, R.; Céspedes, F.; Gabriel, D.; Baeza, M., Microfluidic lab-on-a-chip platforms for environmental monitoring. TrAC Trends in Analytical Chemistry 2017, 95, 62-68.

5. Su, W.; Gao, X.; Jiang, L.; Qin, J., Microfluidic platform towards point-of-care diagnostics in infectious diseases. Journal of Chromatography A 2015, 1377, 13-26.

6. $\quad$ Chen, P.; Chung, M. T.; McHugh, W.; Nidetz, R.; Li, Y.; Fu, J.; Cornell, T. T.; Shanley, T. P.; Kurabayashi, K., Multiplex serum cytokine immunoassay using nanoplasmonic biosensor microarrays. ACS nano 2015, 9 (4), 4173-4181.

7. Zhu, C.; Yang, G.; Li, H.; Du, D.; Lin, Y., Electrochemical sensors and biosensors based on nanomaterials and nanostructures. Analytical chemistry 2015, 87 (1), 230-249.

8. Sechi, D.; Greer, B.; Johnson, J.; Hashemi, N., Three-dimensional paper-based microfluidic device for assays of protein and glucose in urine. Analytical chemistry 2013, 85 (22), 10733-10737.

9. Dou, M.; Dominguez, D. C.; Li, X.; Sanchez, J.; Scott, G., A versatile PDMS/paper hybrid microfluidic platform for sensitive infectious disease diagnosis. Analytical chemistry 2014, 86 (15), 79787986.

10. Altintas, Z.; Akgun, M.; Kokturk, G.; Uludag, Y., A fully automated microfluidic-based electrochemical sensor for real-time bacteria detection. Biosensors and Bioelectronics 2018, 100, 541-548. 11. Mu, X.; Zhang, L.; Chang, S.; Cui, W.; Zheng, Z., Multiplex microfluidic paper-based immunoassay for the diagnosis of hepatitis $C$ virus infection. Analytical chemistry 2014, 86 (11), 5338-5344.

12. Sardesai, N. P.; Kadimisetty, K.; Faria, R.; Rusling, J. F., A microfluidic electrochemiluminescent device for detecting cancer biomarker proteins. Analytical and bioanalytical chemistry 2013, 405 (11), 3831-3838.

13. Jeong, S.; Park, J.; Pathania, D.; Castro, C. M.; Weissleder, R.; Lee, H., Integrated magnetoelectrochemical sensor for exosome analysis. ACS nano 2016, 10 (2), 1802-1809.

14. Xu, M.; Wang, R.; Li, Y., Rapid detection of Escherichia coli O157: H7 and Salmonella Typhimurium in foods using an electrochemical immunosensor based on screen-printed interdigitated microelectrode and immunomagnetic separation. Talanta 2016, 148, 200-208.

15. Shin, S. R.; Zhang, Y. S.; Kim, D.-J.; Manbohi, A.; Avci, H.; Silvestri, A.; Aleman, J.; Hu, N.; Kilic, T.; Keung, W., Aptamer-based microfluidic electrochemical biosensor for monitoring cell-secreted trace cardiac biomarkers. Analytical chemistry 2016, 88 (20), 10019-10027.

16. Guo, X.; Kulkarni, A.; Doepke, A.; Halsall, H. B.; Iyer, S.; Heineman, W. R., Carbohydrate-based label-free detection of Escherichia coli ORN 178 using electrochemical impedance spectroscopy. Analytical chemistry 2012, 84 (1), 241-246.

17. Mohamadi, R. M.; Besant, J. D.; Mepham, A.; Green, B.; Mahmoudian, L.; Gibbs, T.; Ivanov, I.; Malvea, A.; Stojcic, J.; Allan, A. L., Nanoparticle-mediated binning and profiling of heterogeneous circulating tumor cell subpopulations. Angewandte Chemie International Edition 2015, 54 (1), 139-143.

18. Murlidhar, V.; Zeinali, M.; Grabauskiene, S.; Ghannad-Rezaie, M.; Wicha, M. S.; Simeone, D. M.; Ramnath, N.; Reddy, R. M.; Nagrath, S., A radial flow microfluidic device for ultra-high-throughput affinitybased isolation of circulating tumor cells. Small 2014, 10 (23), 4895-4904. 
19. Mao, Z.; Li, P.; Wu, M.; Bachman, H.; Mesyngier, N.; Guo, X.; Liu, S.; Costanzo, F.; Huang, T. J., Enriching nanoparticles via acoustofluidics. ACS nano 2017, 11 (1), 603-612.

20. Hammarström, B.; Laurell, T.; Nilsson, J., Seed particle-enabled acoustic trapping of bacteria and nanoparticles in continuous flow systems. Lab on a Chip 2012, 12 (21), 4296-4304.

21. Mirzajani, H.; Cheng, C.; Wu, J.; Chen, J.; Eda, S.; Aghdam, E. N.; Ghavifekr, H. B., A highly sensitive and specific capacitive aptasensor for rapid and label-free trace analysis of Bisphenol A (BPA) in canned foods. Biosensors and Bioelectronics 2017, 89, 1059-1067.

22. Cheng, I.-F.; Yang, H.-L.; Chung, C.-C.; Chang, H.-C., A rapid electrochemical biosensor based on an AC electrokinetics enhanced immuno-reaction. Analyst 2013, 138 (16), 4656-4662.

23. Li, S.; Cui, H.; Yuan, Q.; Wu, J.; Wadhwa, A.; Eda, S.; Jiang, H., AC electrokinetics-enhanced capacitive immunosensor for point-of-care serodiagnosis of infectious diseases. Biosensors and Bioelectronics 2014, 51, 437-443.

24. Feldman, H. C.; Sigurdson, M.; Meinhart, C. D., AC electrothermal enhancement of heterogeneous assays in microfluidics. Lab on a Chip 2007, 7 (11), 1553-1559.

25. Salari, A.; Thompson, M., Recent advances in AC electrokinetic sample enrichment techniques for biosensor development. Sensors and Actuators B: Chemical 2018, 255, 3601-3615.

26. Ouyang, M.; Mohan, R.; Lu, Y.; Liu, T.; Mach, K. E.; Sin, M. L.; McComb, M.; Joshi, J.; Gau, V.; Wong, P. K., An AC electrokinetics facilitated biosensor cassette for rapid pathogen identification. Analyst 2013, 138 (13), 3660-3666.

27. Cui, H.; Li, S.; Yuan, Q.; Wadhwa, A.; Eda, S.; Chambers, M.; Ashford, R.; Jiang, H.; Wu, J., An AC electrokinetic impedance immunosensor for rapid detection of tuberculosis. Analyst 2013, 138 (23), 7188-7196.

28. Li, S.; Ren, Y.; Jiang, H., Convection and mass transfer enhanced rapid capacitive serum immunoassay. Rsc Advances 2014, 4 (18), 9064-9071.

29. Liu, X.; Yang, K.; Wadhwa, A.; Eda, S.; Li, S.; Wu, J., Development of an AC electrokinetics-based immunoassay system for on-site serodiagnosis of infectious diseases. Sensors and Actuators A: Physical 2011, 171 (2), 406-413.

30. Hart, R.; Ergezen, E.; Lec, R., Improved protein detection on an AC electrokinetic quartz crystal microbalance (EKQCM). Biosensors and Bioelectronics 2011, 26 (8), 3391-3397.

31. Sin, M. L.; Liu, T.; Pyne, J. D.; Gau, V.; Liao, J. C.; Wong, P. K., In situ electrokinetic enhancement for self-assembled-monolayer-based electrochemical biosensing. Analytical chemistry 2012, 84 (6), 27022707.

32. Liu, X.; Cheng, C.; Wu, J.; Eda, S.; Guo, Y., A low cost and palm-size analyzer for rapid and sensitive protein detection by AC electrokinetics capacitive sensing. Biosensors and Bioelectronics 2017, 90, 83-90.

33. Cui, H.; Cheng, C.; Lin, X.; Wu, J.; Chen, J.; Eda, S.; Yuan, Q., Rapid and sensitive detection of small biomolecule by capacitive sensing and low field AC electrothermal effect. Sensors and Actuators $B$ : Chemical 2016, 226, 245-253.

34. Mansoorifar, A.; Koklu, A.; Ma, S.; Raj, G. V.; Beskok, A., Electrical impedance measurements of biological cells in response to external stimuli. Analytical chemistry 2018, 90 (7), 4320-4327.

35. Fernandez, R. E.; Koklu, A.; Mansoorifar, A.; Beskok, A., Platinum black electrodeposited thread based electrodes for dielectrophoretic assembly of microparticles. Biomicrofluidics 2016, 10 (3), 033101.

36. Mansoorifar, A.; Koklu, A.; Sabuncu, A. C.; Beskok, A., Dielectrophoresis assisted loading and unloading of microwells for impedance spectroscopy. Electrophoresis 2017, 38 (11), 1466-1474.

37. Song, Y.; Chen, P.; Chung, M. T.; Nidetz, R.; Park, Y.; Liu, Z.; McHugh, W.; Cornell, T. T.; Fu, J.; Kurabayashi, K., AC electroosmosis-enhanced nanoplasmofluidic detection of ultralow-concentration cytokine. Nano letters 2017, 17 (4), 2374-2380.

38. Koklu, A.; Tansel, O.; Oksuzoglu, H.; Sabuncu, A. C., Electrothermal flow on electrodes arrays at physiological conductivities. IET nanobiotechnology 2016, 10 (2), 54-61. 
39. Castellanos, A.; Ramos, A.; Gonzalez, A.; Green, N. G.; Morgan, H., Electrohydrodynamics and dielectrophoresis in microsystems: scaling laws. Journal of Physics D: Applied Physics 2003, 36 (20), 2584. 40. Park, S.; Beskok, A., Alternating current electrokinetic motion of colloidal particles on interdigitated microelectrodes. Analytical Chemistry 2008, 80 (8), 2832-2841.

41. Koklu, A.; Sabuncu, A. C.; Beskok, A., Enhancement of dielectrophoresis using fractal gold nanostructured electrodes. Electrophoresis 2017, 38 (11), 1458-1465.

42. Salari, A.; Navi, M.; Lijnse, T.; Dalton, C., AC Electrothermal Effect in Microfluidics: A Review. Micromachines 2019, 10 (11), 762.

43. Koklu, A.; El Helou, A.; Raad, P. E.; Beskok, A., Characterization of Temperature Rise in Alternating Current Electrothermal Flow Using Thermoreflectance Method. Analytical chemistry 2019, 91 (19), 1249212500.

44. Park, S.; Koklu, M.; BeskoK, A., Particle trapping in high-conductivity media with electrothermally enhanced negative dielectrophoresis. Analytical chemistry 2009, 81 (6), 2303-2310.

45. Amatore, C.; Fosset, B.; Bartelt, J.; Deakin, M.; Wightman, R., Electrochemical kinetics at microelectrodes: Part V. Migrational effects on steady or quasi-steady-state voltammograms. Journal of electroanalytical chemistry and interfacial electrochemistry 1988, 256 (2), 255-268.

46. Ciszkowska, M.; Stojek, Z., Voltammetry in solutions of low ionic strength. Electrochemical and analytical aspects. Journal of Electroanalytical Chemistry 1999, 466 (2), 129-143.

47. Yuan, J.; Wang, K.; Xia, X., Highly ordered platinum-nanotubule arrays for amperometric glucose sensing. Advanced Functional Materials 2005, 15 (5), 803-809.

48. Wei, A.; Sun, X. W.; Wang, J.; Lei, Y.; Cai, X.; Li, C. M.; Dong, Z.; Huang, W., Enzymatic glucose biosensor based on $\mathrm{ZnO}$ nanorod array grown by hydrothermal decomposition. Applied Physics Letters 2006, 89 (12), 123902.

49. Luo, D.; Wu, L.; Zhi, J., Fabrication of boron-doped diamond nanorod forest electrodes and their application in nonenzymatic amperometric glucose biosensing. Acs Nano 2009, 3 (8), 2121-2128.

50. Lee, S.-W.; Lee, K.-S.; Ahn, J.; Lee, J.-J.; Kim, M.-G.; Shin, Y.-B., Highly sensitive biosensing using arrays of plasmonic Au nanodisks realized by nanoimprint lithography. ACS nano 2011, 5 (2), 897-904.

51. Koklu, A.; Ajaev, V.; Beskok, A., Self-Similar Response of Electrode Polarization for Binary Electrolytes in Parallel Plate Capacitor Systems. Analytical chemistry 2019, 91 (17), 11231-11239.

52. Koklu, A.; Mansoorifar, A.; Beskok, A., Effects of electrode size and surface morphology on electrode polarization in physiological buffers. Electrophoresis 2019, 40 (5), 766-775.

53. Koklu, A.; Sabuncu, A. C.; Beskok, A., Rough gold electrodes for decreasing impedance at the electrolyte/electrode interface. Electrochimica acta 2016, 205, 215-225.

54. Koklu, A.; Mansoorifar, A.; Beskok, A., Self-similar interfacial impedance of electrodes in high conductivity media. Analytical chemistry 2017, 89 (22), 12533-12540.

55. Koklu, A.; Mansoorifar, A.; Giuliani, J.; Monton, C.; Beskok, A., Self-Similar Interfacial Impedance of Electrodes in High Conductivity Media: II. Disk Electrodes. Analytical chemistry 2018, 91 (3), 2455-2463. 56. Guiliani, J.; Cadena, J.; Monton, C., Template-assisted electrodeposition of $\mathrm{Ni}$ and $\mathrm{Ni} / \mathrm{Au}$ nanowires on planar and curved substrates. Nanotechnology 2018, 29 (7), 075301.

57. Lee, W. C.; Lee, H.; Lim, J.; Park, Y. J., An effective electrical sensing scheme using AC electrothermal flow on a biosensor platform based on a carbon nanotube network. Applied Physics Letters 2016, 109 (22), 223701.

58. San Vicente, G.; Bayón, R.; Germán, N.; Morales, A., Surface modification of porous antireflective coatings for solar glass covers. Solar Energy 2011, 85 (4), 676-680.

59. Terpilowski, K.; Goncharuk, O., Hydrophobic properties of hexamethyldisilazane modified nanostructured silica films on glass: effect of plasma pre-treatment of glass and polycondensation features. Materials Research Express 2018, 5 (1), 016409. 
60. Wang, F.; Wang, X.; Xie, A.; Shen, Y.; Duan, W.; Zhang, Y.; Li, J., A simple method for preparation of transparent hydrophobic silica-based coatings on different substrates. Applied Physics A 2012, 106 (1), 229-235.

61. Pecheva, E. In Study of Biocompatible and Biological Materials: Can they be influenced by external factors?, Materials Research Forum LLC: 2017.

62. Ayibaike, D.; Cui, M.; Wei, J., Single-Cell Patterning Based on Immunocapture and a Surface Modified Substrate. Applied Sciences 2018, 8 (11), 2152.

63. Feng, X.; Feng, L.; Jin, M.; Zhai, J.; Jiang, L.; Zhu, D., Reversible super-hydrophobicity to superhydrophilicity transition of aligned ZnO nanorod films. Journal of the American Chemical Society 2004, 126 (1), 62-63.

64. Ramos, A.; Morgan, H.; Green, N. G.; Castellanos, A., Ac electrokinetics: a review of forces in microelectrode structures. Journal of Physics D: Applied Physics 1998, 31 (18), 2338.

65. Green, N. G.; Ramos, A.; Gonzalez, A.; Castellanos, A.; Morgan, H., Electrothermally induced fluid flow on microelectrodes. Journal of Electrostatics 2001, 53 (2), 71-87.

66. Liu, T.; Sin, M. L.; Pyne, J. D.; Gau, V.; Liao, J. C.; Wong, P. K., Electrokinetic stringency control in self-assembled monolayer-based biosensors for multiplex urinary tract infection diagnosis. Nanomedicine: Nanotechnology, Biology and Medicine 2014, 10 (1), 159-166.

67. Chaurey, V.; Polanco, C.; Chou, C.-F.; Swami, N. S., Floating-electrode enhanced constriction dielectrophoresis for biomolecular trapping in physiological media of high conductivity. Biomicrofluidics 2012, 6 (1), 012806. 

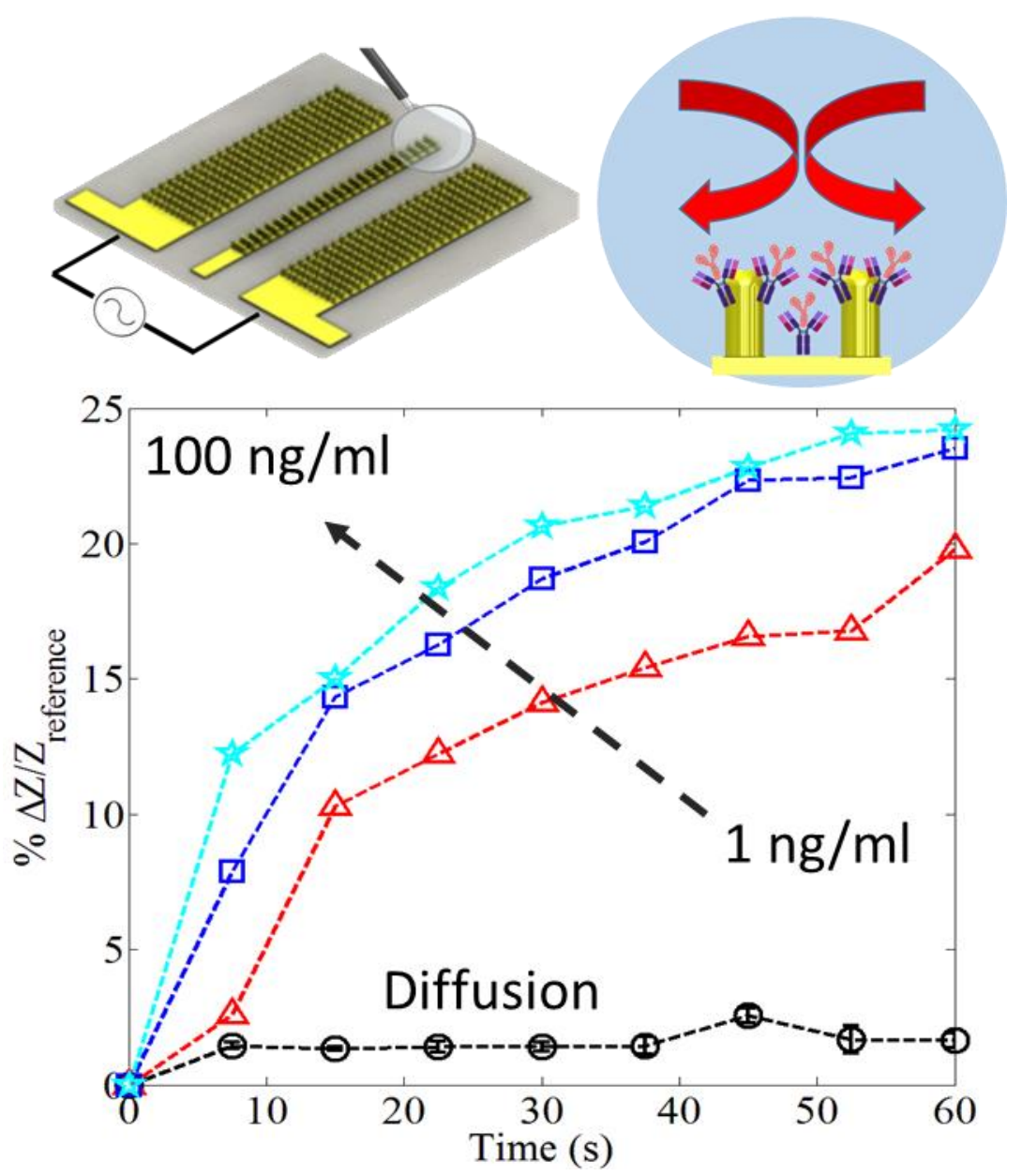

For Table of Contents Only 